\title{
Costing pressure ulcer care in an Irish acute care setting: a feasibility study
}

Objective: To test the feasibility of using a standardised data collection tool to estimate the cost of stage 2-4 pressure ulcer (PU) care within an acute care setting.

Method: Data on resource use and cost were obtained through a retrospective survey of nursing and medical notes collecting cost data for individual patients who received care for stage 2-4 PUs. Results: Data for 20 patients (12 male/ 8 female) were analysed. The average patient age was 69 years (range: $37-95$ years). Of this sample, seven patients had hospital-acquired PUs (HAPUs) and 14 patients had community-acquired PUs (CAPU) (one patient had both-in different anatomical areas). Over half of the total sample (55\%; $n=11)$ had a stage $2 \mathrm{PU}$. The average length of stay was 31.8 days (range: $5-119$ days). Most of the patients $(70 \% ; n=14)$ had a CAPU. The average cost per patient with PU care was $€ 878$ (range:
$€ 39-2393)$. The mean cost for patients with a HAPU was €866 (SD: $€ 1313$ ) versus $€ 911$ (SD: $€ 567)$ for patients with a CAPU. The majority of the cost related to equipment and staff time for treatment. Conclusion: Overall, the application of the standardised data collection tool to obtain cost data from retrospective inspection of nursing and medical notes is feasible. The cost of PU care in this sample was high, indicating that these wounds may impose a substantial burden on health systems. The costs varied greatly between patients in the sample, reflecting the complexity of PU care. Furthermore, given that costs increased with the higher PU stages, there is a potential to reduce costs by preventing the development of higher stage PUs. Larger-scale studies are required to understand the cost variation and full economic impact of PU care.

Declaration of interest: The authors have no conflicts of interest.

acute care setting • community-acquired pressure ulcer $\bullet$ cost of pressure ulcers $\bullet$ dressing $\bullet$ hospital-acquired pressure ulcer $\bullet$ pressure ulcer $\bullet$ standardised data collection tool $\bullet$ wound $\bullet$ wounds $\bullet$ wound treatment

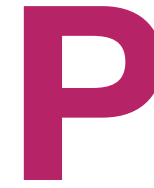
ressure ulcers (PUs) are a problem in all healthcare settings and are defined as localised damage to the skin and/or underlying tissue, as a result of pressure or pressure in combination with shear. ${ }^{1}$ The prevalence of PU ranges from $0-72.5 \%$ within different clinical settings. ${ }^{2-5}$ A recent systematic review reported a European median PU prevalence rate of $10.8 \%$ among hospitalised patients (standard deviation (SD): 7\%; range: $4.6-27.2 \%){ }^{6}$

PUs are often difficult to heal, painful and impact

Aoife Reilly, ${ }^{1}$ Medical Student; Jan Sorensen, ${ }^{2}$ Professor; Helen Strapp ${ }^{3}$;

Declan Patton, ${ }^{4,5,6,7} \mathrm{PhD}$, Director of Nursing and Midwifery Research and Deputy Director of the Skin, Wounds and Trauma Research Centre; Amy Blair, ${ }^{1}$ Medical Student; Pinar Avsar ${ }^{4}$, PhD*; Jane Burns $^{8}$; Zena Moore ${ }^{4,9,10,11,12,13,14}$ Professor of Nursing, Head of School of Nursing and Midwifery and Director of the Skin Wounds and Trauma Research Centre.

Corresponding author email: pinaravsar@rcsi.com

1 Royal College of Surgeons in Ireland. 2 Healthcare Outcomes Research Centre, Royal College of Surgeons in Ireland. 3 Tallaght Hospital, Ireland. 4 School of Nursing and Midwifery, Royal College of Surgeons in Ireland University of Medicine and Health Sciences, Dublin. 5 Adjunct Associate Professor, Fakeeh College of Health Sciences, Jeddah, Saudi Arabia. 6 Honorary Senior Fellow, Faculty of Science, Medicine and Health, University of Wollongong, Australia. 7 Adjunct Professor, Griffith University, Australia. 8 Athlone Institute of Technology, Ireland. 9 Adjunct Professor, Fakeeh College of Health Sciences, Jeddah, Saudi Arabia. 10 Adjunct Professor, Faculty of Medicine, Nursing and Health Sciences, Monash University, Australia. 11 Professor, Department of Public Health, Faculty of Medicine and Health Sciences, Ghent University, Belgium. 12 Honorary Professor, Lida Institute, Shanghai, China. 13 Visiting Professor, University of Wales, Cardiff, UK. 14 Adjunct Professor, Griffith University, Australia. negatively on the individual's quality of life. ${ }^{7}$ The cost implications of PU treatment are considerable, compounding the challenges of providing cost-effective, efficient health service delivery. ${ }^{8-10}$ In a systematic review, the cost of PU treatment per patient per day was estimated to range between $€ 1.71$ and $€ 470.49$ across all settings. ${ }^{11}$ Meanwhile, in Ireland, it has been estimated that PU management costs $€ 250$ million per annum across all care settings. ${ }^{10}$ The total cost of PU care in the UK is greater again, where it has been reported as costing $£ 1.4-£ 2.1$ billion, which accounts for $4 \%$ of total NHS expenditure. ${ }^{12}$

Furthermore, in the US, Allison et al. ${ }^{13}$ reported that although hospital stays principally for PUs were longer than hospital stays with a secondary diagnosis of PUs and those with no PU diagnosis, the average cost per day (\$1200) was lower-nearly \$400 less than secondary PU hospital stays ( $\$ 1600$ per day) and $\$ 800$ less than hospitals stays for all other conditions ( $\$ 2000$ per day).

Economic evaluations compare the cost and effects of alternative interventions or programmes, and aim to help decision-makers to identify the most cost-effective interventions for a particular group of patients. ${ }^{14}$ It is common to restrict the focus of the analysis by including the use of healthcare resource within a defined period of time (for example, while admitted at hospital). When the health effects of the programmes are similar, cost-minimisation analysis compares the resource use and cost of the interventions. Costs are usually determined through measures of resource use multiplied 
by relevant unit costs. This requires a careful measurement of the actual resources used in the programme. Such measurement can be based on direct observation or through inspection of records of actual resource use.

Several studies have provided insight into the economic burden of PUs for society, patients, health services and insurers. These studies have been conducted across several settings and countries, using different approaches and methods, with the quality of reporting varying widely. ${ }^{10-12}$ To date, no studies within the Irish health service have explored the cost of managing PUs of all stages and the exact economic impact of PUs remains largely unknown. Given the significant prevalence and incidence of PUs, it is important to obtain greater insight into the economic impact. The aim of this study was to test the feasibility of using a standardised data collection tool to estimate the cost of stage 2-4 PU care within an acute care setting.

\section{Method}

A retrospective survey design was employed to obtain data on resource use and unit costs to test the feasibility of using a standardised data collection tool to estimate the cost of PU care. This involved collecting relevant data on the care of patients with stage 2-4 PUs from the nursing and medical (paper-based) notes of 20 participants admitted to an acute Irish hospital from February to July 2017. The hospital had 634 beds, providing child health, adult, psychiatric and agerelated healthcare on one site. The PU rate at the time of the study was $24 \%$. The data were collected by one of the authors and the tissue viability nurse reviewed it for accuracy. The tissue viability nurses kept a record of patients who had developed stage 2-4 PUs in the preceding five months before the data collection began, and these patients formed the basis of the sample.

The choice of data to collect was guided by an international consensus document. ${ }^{15}$

The data collection tool was developed based on literature and expert opinion ${ }^{8-10}$ and included three parts. The first addressed demographic characteristics of the patients such as age, sex, length of stay and mobility status. The second part included the European Pressure Ulcer Advisory Panel (EPUAP) PU classification system, a six-stage system to classify PUs. The stages are: ${ }^{16}$

1. Nonblanchable erythema: stage 1

2. Partial-thickness skin loss: stage 2

3. Full-thickness skin loss: stage 3

4. Full-thickness tissue loss: stage 4

5. Depth unknown: unstageable

6. Suspected deep tissue injury: depth unknown.

The third part of the data collection tool included cost items. These items $(n=81)$ were categorised into four main cost topics (which were selected by the health economist):

1. Equipment (heel boots, pressure relieving mattresses)

2. Materials (creams, dressings, drugs)

3. Tests

4. Nurse time (repositioning).
Table 1. Demographic characteristics of patients

\begin{tabular}{|c|c|c|}
\hline Descriptive characteristics & $\mathbf{n}$ & $\%$ \\
\hline \multicolumn{3}{|l|}{ Sex } \\
\hline Female & 8 & 40.0 \\
\hline Male & 12 & 60.0 \\
\hline \multicolumn{3}{|l|}{ Primary diagnosis } \\
\hline Respiratory tract infection & 2 & 10 \\
\hline Urinary tract infection & 2 & 10 \\
\hline Lower limb fracture & 4 & 20 \\
\hline Neurological & 1 & 5 \\
\hline Psychiatric & 1 & 5 \\
\hline Pressure ulcer deterioration & 2 & 10 \\
\hline Sepsis & 6 & 30 \\
\hline Gastrointestinal & 2 & 10 \\
\hline \multicolumn{3}{|c|}{$\begin{array}{l}\text { Age: Mean: } 68.95 \text { years; standard deviation (SD): } 15.7 \text { years; } \\
\text { range: } 37-95 \text { years }\end{array}$} \\
\hline
\end{tabular}

Table 2. Stages of pressure ulcers

\begin{tabular}{|c|c|c|}
\hline Pressure ulcer stage & $\mathbf{n}$ & $\%$ \\
\hline Stage 2 & 11 & 55.0 \\
\hline Stage 3 & 2 & 10.0 \\
\hline Stage 4 & 7 & 35.0 \\
\hline
\end{tabular}

The unit cost of each item/service was obtained from the hospital's procurement department. The cost per patient was calculated as the accumulated sum of the actual use of resources multiplied by the unit cost. Cost of nurse time was calculated based on the monthly gross salary of an experienced ward nurse and divided by the number of effective working hours in a month (i.e., excluding time related to leave, sickness and other absence, and non-patient-related tasks).

All data were analysed using the statistical software package, Statistical Analysis System (SAS) version 9.2. Frequency, percentage, mean and SD were calculated for evaluations of the data. As it was part of a clinical/ economic audit and the patients could not be identified in the data material, no formal consent of patients was required. Permission to undertake the research was obtained from the hospital governance committee.

\section{Data collection tool}

The tool included the following items:

- Equipment (mattress type, peripherally inserted central catheter (PICC line), heel boots, cushion)

- Tests (swab, bloods, magnetic resonance imaging (MRI), biopsy, X-ray, ankle-brachial pressure index (ABPI), toe pressures) 


\section{practice}

Table 3. Treatment direct cost per patient (2018; euros)

\begin{tabular}{|c|c|c|c|c|c|c|}
\hline \multirow[t]{2}{*}{ Cost items } & \multicolumn{2}{|c|}{$\begin{array}{c}\text { Stage } 2 \\
n=11\end{array}$} & \multicolumn{2}{|c|}{$\begin{array}{c}\text { Stage } 3 \\
n=2\end{array}$} & \multicolumn{2}{|c|}{$\begin{array}{c}\text { Stage } 4 \\
n=7\end{array}$} \\
\hline & Mean cost & $\%$ & Mean cost & $\%$ & Mean cost & $\%$ \\
\hline $\begin{array}{l}\text { Equipment (e.g., mattress, } \\
\text { heel boots) }\end{array}$ & 1124.18 & 51.0 & 2151.5 & 47.7 & 458.8 & 38.7 \\
\hline $\begin{array}{l}\text { Tests (i.e., swab, bloods, MRI, } \\
\text { biopsy, X-ray, ABPI, toe pressures) }\end{array}$ & 48 & 2.1 & - & - & 118 & 9.8 \\
\hline Repositioning & 49.63 & 2.2 & 52 & 1.1 & 47.33 & 3.9 \\
\hline Dressings & 35.55 & 1.6 & 64.63 & 1.4 & 24.53 & 2.0 \\
\hline Ointments & 7.64 & 0.3 & 7.32 & 0.1 & 8.54 & 0.7 \\
\hline $\begin{array}{l}\text { Medication (i.e.,antibiotics, } \\
\text { analgesics, other medication used } \\
\text { just for treating pressure ulcers (PUs)) }\end{array}$ & 13.5 & 0.6 & - & - & 13.5 & 1.1 \\
\hline Supplements & - & - & - & - & 21.29 & 1.7 \\
\hline Treating staff for PU specifically & 920.48 & 42.2 & 2256.49 & 49.7 & 503.80 & 42.1 \\
\hline Average cost per patient & & & & & & \\
\hline \multicolumn{7}{|l|}{ The total cost of care $(n=20): € 17,550$} \\
\hline \multicolumn{7}{|c|}{ Mean cost per patient: €877.5; standard deviation: €193.3 (minimum: €38.5; maximum: €2392.8) } \\
\hline Mean length of stay: 31.8 days; standa & leviation: 6.7 & imum: & aximum: 119 & de: 7.0 & dian: $20.0 \mathrm{da}$ & \\
\hline
\end{tabular}

- Intervention (repositioning, number of staff, debridement, dressings, negative wound pressure therapy (NPWT), ointments, medication, supplements and staff treating PU specifically).

\section{Results}

\section{Demographic data}

The sample population comprised 20 patients. The demographic characteristics of patients are summarised in Table 1 . The age of patients varied from 37-95 years old, with a mean age of 69 years (SD: 16 years). In relation to sex, $60 \%(n=12)$ were female and $40 \%(n=8)$ were male. Of the patients, six (30\%) had a primary diagnosis of sepsis (Table 1). Most of the patients (70\%; $\mathrm{n}=14$ ) had a community-acquired PU. Over half of the total sample $(55 \% ; \mathrm{n}=11)$ had a stage $2 \mathrm{PU}$ (Table 2$)$.

\section{Cost results}

The findings on the direct costs of PU treatment showed that the total cost of treatment for the 20 patients was $€ 17,550$. The mean cost per PU was $€ 878$ (SD: $€ 193$ range: €39-2393) per patient. The mean length of stay was 31.8 days (SD: 6.7 days; range: 5-119; mode: 7.0; median: 20.0) (Table 3).

Costs increased as the severity of the PU increased (i.e., stage 2 versus stages 3 and 4 ). The average cost per patient was €2199 (11 patients) for stage 2 PUs, €4532 (two patients) for stage 3 PUs and $€ 1196$ (seven patients) for stage 4 PUs. Equipment such as a mattress and heel boots accounted for the majority of treatment costs (51\%), followed by staff for the specific treatment of stage 2 PUs (42.2\%). Staff for the specific treatment of stage 3 and 4 PUs accounted for slightly less than half of the treatment cost ( $49.7 \%$ and $42.1 \%$, respectively), followed by equipment ( $47.7 \%$ and $38.7 \%$, respectively) (Table 3).

No statistically significant relationship could be found between the cost and patient characteristics.

\section{Discussion}

This study set out to test the feasibility of using a standardised data collection tool to estimate the cost of PU care. The developed tool, which focused on four main resource components (equipment, materials, tests and nurse time) provided a structured method to obtain data on resource use from nursing and medical records. The costs of PU treatment ranged from $€ 39$ to $€ 2393$ per patient. The average cost related to PU treatment for patients with stage 2 PUs was €2199, $€ 4532$ for patients with stage 3 PUs and €1196 for patients with stage 4 PUs. Demarré et al. ${ }^{9}$ investigated the cost of PU treatment and prevention in Belgium, and determined that the treatment cost ranged between $€ 2.34$ and $€ 77.36$. Schuurman et al. ${ }^{17}$ studied 250 patients and calculated the average daily cost per patient at $€ 37$. In addition, Dealey et al. ${ }^{18}$ estimated the treatment costs of PUs from $£ 1214$ (stage 1) to $£ 14,108$ (stage 4) in the UK. In Ireland, Gethin et al. ${ }^{10}$ determined 
the mean cost of management of PUs across all care settings at $€ 480,000$ per patient per annum $(\mathrm{n}=519$ patients). This discrepancy in costs within the literature and in this study may be associated with the variations in the used methods, costing perspectives, study setting (hospitals or nursing homes), country of the study, treatment methods, hospital policies, drugs and nursing costs. Furthermore, estimating the total cost of a PU in isolation will always be complex, given that the majority of patients studied will have at least a dual diagnosis, for which the costs of care are difficult to delineate from those directly attributable to PU treatment.

According to the literature, the majority of the cost attributable to PU care relates to nursing interventions for the treatment of PUs. ${ }^{9,12,17,19}$ In this study, the majority of the cost involved in PU treatment related to the use of equipment and staff for treating PUs specifically.

In the current study, the majority of the participants had a stage 2 PU; however, an increasing PU stage was associated with increased costs. The costs of stage 4 PUs and related complications may be extremely high. ${ }^{20}$ In contrast, according to the present research, the costs of stage 3 PUs were high. This difference in these findings may be related to the design of the study. It must be noted that almost half of the cost associated with stage 3 PUs in this study was attributable to equipment procurement. The cost of equipment in the stage $4 \mathrm{PU}$ cohort was substantially less. Only new equipment ordered during the patient's time in hospital was calculated as cost in this study. This discrepancy may be explained by the chronic nature of PUs, meaning patients with stage 4 PUs may have already had previously acquired equipment, the cost of which was not captured by this study.

Retrospective studies may suggest less effort in analysing large amounts of data. However, retrospective data collection is limited by the accuracy and comprehensiveness of previously recorded data. ${ }^{21}$ If the review of medical and nursing records cannot be captured effectively, it may cause the study to miss variables of interest and fail to adequately reflect specific interventions within the retrospective cohort. ${ }^{21}$ In this study, data collection was challenging. It was difficult to collect the necessary costs, as current inpatient charts were inconsistent and often absent. These findings further support the idea of prospectively designed studies, which require significant financial input but collect more valid and reliable data. Ideally, future studies should address the complexity of gathering health economic data and ensure that a robust data collection process is planned and executed.

\section{Limitations}

The generalisability of these results is subject to certain limitations. For instance, costs not assessed in this study include the costs associated with PU prevention; and costs avoided by PU prevention and indirect societal costs associated with PUs such as lost productivity-

Data collection proved difficult for this project, which questions the feasibility of a retrospective cost analysis of this kind. Firstly, obtaining accurate details regarding the unit cost of products, for instance the cost of each piece of pressure-relieving equipment, was met with resistance from hospital procurement departments due to concerns about sharing supplier prices. Unit costs had to be requested individually and often took weeks to be sent back, frequently with several omissions. Secondly, the level of detail documented in medical charts was highly variable, with some staff documenting exact quantities of materials used and others estimating or providing no such details regarding quantity of products used. This meant that despite the use of the cost analysis tool, the accuracy of the results cannot be determined.

\section{Conclusion}

The present study was designed as a feasibility study of using a standardised data collection tool to estimate the cost of PU care. It demonstrated that relevant data on resource use can be obtained through systematic inspection of nursing and medical records. Overall, the cost of treating PUs in this sample was high, highlighting the considerable economic burden of PUs on health systems. The cost of managing PUs varied greatly between patients, indicating the complexity of PU care and economic analysis but also the potential to reduce costs by preventing development of a higher PU stage. The data collection proved difficult due to lack of detail and inconsistency in documentation in patient charts and difficulty accessing unit costs. To provide a more exact cost analysis of PU care, a larger prospective study could be conducted using the proposed data collection tool. Recognising the significant costs of the problem for both patients and the health and social care system will encourage greater involvement of all health professionals. Jwc

\section{References}

1 European Pressure Ulcer Advisory Panel, National Pressure Injury Advisory Panel, Pan Pacific Pressure Injury Alliance. Prevention and treatment of pressure ulcers/injuries: clinical practice guideline. 2019. http://www.internationalguideline.com (accessed 3 March 2020) 2 Hiser B, Rochette J, Philbin S et al. Implementing a pressure ulcer prevention program and enhancing the role of the CWOCN: impact on outcomes. Ostomy Wound Manage 2006; 52(2):48-59
Reflective questions

- Why is it important to present financial input for pressure ulcers (PUs)?

- How can a standardised data collection tool be used for estimating the cost of PU care?

What are the challenges for estimating the cost of PU care? 


\section{practice}

3 Samaniego IA. A sore spot in pediatrics: risk factors for pressure ulcers. Pediatr Nurs 2003; 29(4):278-282

4 Tubaishat A, Papanikolaou P, Anthony D, Habiballah L. Pressure ulcers prevalence in the acute care setting: a systematic review, 2000-2015. Clin Nurs Res 2018; 27(6):643-659. https://doi.

org/10.1177/1054773817705541

5 Almutairi K, Hendrie D. Global incidence and prevalence of pressure injuries in public hospitals: a systematic review. Wound Medicine 2018; 22:23-31. https://doi.org/10.1016/j.wndm.2018.05.004

6 Moore Z, Avsar P, Conaty L et al. The prevalence of pressure ulcers in Europe, what does the European data tell us: a systematic review. J Wound Care 2019; 28(11):710-719. https://doi.org/10.12968/ jowc.2019.28.11.710

7 Gorecki C, Brown JM, Nelson EA et al.; European Quality of Life Pressure Ulcer Project group. Impact of pressure ulcers on quality of life in older patients: a systematic review. J Am Geriatr Soc 2009; 57(7):11751183. https://doi.org/10.1111/j.1532-5415.2009.02307.x

8 Moore Z, Cowman S, Posnett J. An economic analysis of repositioning for the prevention of pressure ulcers. J Clin Nurs 2013; 22(15-16):23542360. https://doi.org/10.1111/j.1365-2702.2012.04310.x

9 Demarré L, Verhaeghe S, Annemans L et al. The cost of pressure ulcer prevention and treatment in hospitals and nursing homes in Flanders: a cost-of-illness study. Int J Nurs Stud 2015; 52(7):1166-1179. https://doi. org/10.1016/j.jinurstu.2015.03.005

10 Gethin G, Jordan-O'Brien J, Moore Z. Estimating costs of pressure area management based on a survey of ulcer care in one Irish hospital. J Wound Care 2005; 14(4):162-165. https://doi.org/10.12968/ jowc.2005.14.4.26759

11 Demarré L, Van Lancker A, Van Hecke A et al. The cost of prevention and treatment of pressure ulcers: a systematic review. Int J Nurs Stud 2015; 52(11):1754-1774. https://doi.org/10.1016/j.ijnurstu.2015.06.006 12 Bennett G, Dealey C, Posnett J. The cost of pressure ulcers in the UK.
Age Ageing 2004; 33(3):230-235. https://doi.org/10.1093/ageing/afh086 13 Russo CA, Steiner C, Spector W. Hospitalizations related to pressure ulcers among adults 18 years and older, 2006. Healthcare Cost and Utilization Project. https://www.hcup-us.ahrq.gov/reports/statbriefs/sb64 pdf (accessed 3 March 2020)

14 Drummond MF Sculpher MJ, Torrance GW et al. Methods for the economic evaluation of health care programme. Third edition. Oxford: Oxford University Press, 2005

15 International Consensus. Making the case for cost-effective wound management. Wounds International 2013. www.woundsinternational.com 16 National Pressure Ulcer Advisory Panel, European Pressure Ulcer Advisory Panel and Pan Pacific Pressure Injury Alliance. Prevention and treatment of pressure ulcers: quick reference guide. 2014. https://tinyurl. com/afzkvd2e (accessed 3 March 2020)

17 Schuurman JP, Schoonhoven L, Defloor T et al. Economic evaluation of pressure ulcer care: a cost minimization analysis of preventive strategies. Nurs Econ 2009; 27(6):390-400

18 Dealey C, Posnett J, Walker A. The cost of pressure ulcers in the United Kingdom. J Wound Care 2012; 21(6):261-266. https://doi. org/10.12968/jowc.2012.21.6.261

19 Avsar P, Karadag A. Efficacy and cost-effectiveness analysis of evidence-based nursing interventions to maintain tissue integrity to prevent pressure ulcers and incontinence-associated dermatitis.

Worldviews Evid Based Nurs 2018; 15(1):54-61. https://doi.org/10.1111/ wvn. 12264

20 Brem H, Maggi J, Nierman D et al. High cost of stage IV pressure ulcers. Am J Surg 2010; 200(4):473-477. https://doi.org/10.1016/j. amjsurg.2009.12.021

21 Clark M. Retrospective versus prospective cohort study designs for evaluating treatment of pressure ulcers: a comparison of 2 studies. J Wound Ostomy Continence Nurs 2008; 35(4):391-394. https://doi. org/10.1097/01.WON.0000326657.57939.c9

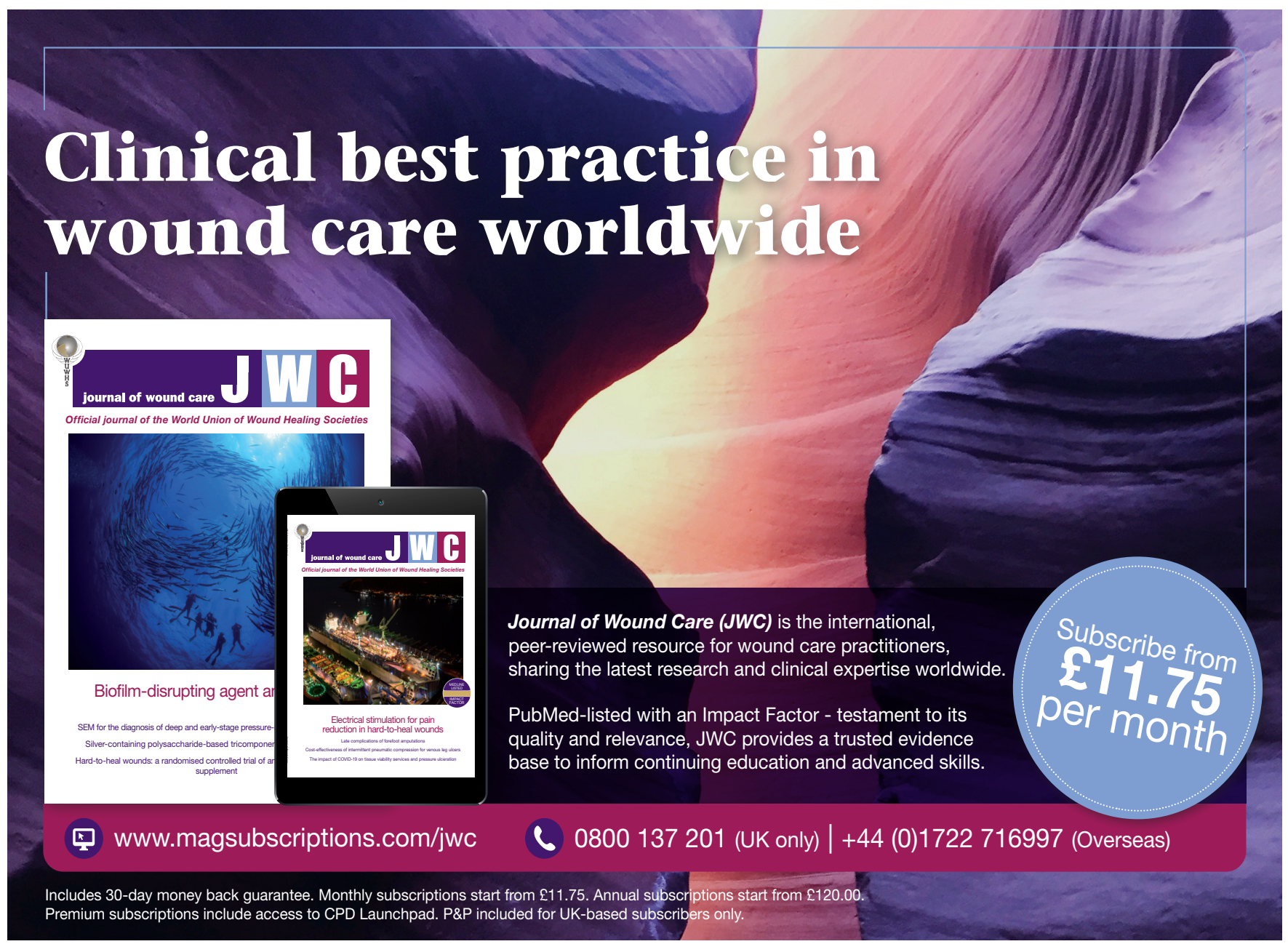

